\title{
On Reconciliation of Contractual Concerns of Web Services $^{\star}$
}

\author{
Hong-Linh Truong ${ }^{1}$, G.R. Gangadharan ${ }^{2}$, Martin Treiber ${ }^{1}$, Schahram Dustdar ${ }^{1}$, \\ Vincenzo D'Andrea ${ }^{3}$ \\ 1 Distributed Systems Group, Vienna University of Technology \\ \{truong, treiber, dustdar\}einfosys.tuwien.ac.at \\ 2 Telematica Institute, The Netherlands \\ gretelin.nl \\ 3 Department of Information Technology, University of Trento \\ dandrea@dit.unitn.it
}

\begin{abstract}
Contracts are a way of collaborating and binding parties involved describing exchanges of promises or agreement between parties. In the world of Web services, the approaches, languages, and tools that support, specify, and establish contractual concerns are vast. Existing works often mention similar concepts but provide different languages and terminologies. In most cases, current works do not consider the negotiation and establishment of contractual terms modeled by different specifications. In particular, the issue of interoperability among different Web service contract frameworks is open. We analyze existing approaches and propose guidelines for reconciling them in order to improve collaboration between service providers, service consumers and aggregators.
\end{abstract}

\section{Introduction}

Contractual concerns of services refer to functional/non-functional properties and business/management information of services. A service contract ${ }^{1}$ associated with a Web service describes how the usage of a service should be, for example, whether a service is free for non-commercial purposes. A service contract includes normative aspects ${ }^{2}$ that are agreed between the service consumer and the service provider who acted in compliance with the contract. A service contract is a complicated artifact surrounded with interdisciplinary research efforts. As a result, various terminologies, specifications, and tools have been developed. Unfortunately, many of them address similar problems but speak in different languages and are not interoperable.

There exist many works addressing, fully or partial, one or multiple phases of service contracts, but they often mention the same thing with different languages and terminologies. This requires guidelines and approaches for reconciling them to better collaboration between service providers, consumers and aggregators. In most cases, current works do not consider the negotiation and establishment of contractual terms modeled

\footnotetext{
* The work mentioned in this paper is partially funded by the FP7 COMPAS and COIN projects.

${ }^{1}$ In this paper, we discuss service contracts at operational level, not business level [1]

2 http://en.wikipedia.org/wiki/Normative
} 
by different specifications. However, such negotiation and establishment should be supported as service consumers are increasingly composing and utilizing services provided by different organizations, each imposes a different meaning on contractual terms. In parallel, the trend of providing software as a service (SaaS) demands a strong support for establishment, monitoring and enforcement of diverse contractual terms as in SaaS model typically each software usage is bound to a service contract. Addressing service contracts for composite services is important but this work is under-researched.

In this paper, we analyze the complexity of current contractual concerns, identify issues preventing the establishment of contractual concerns in the service composition, and propose guidelines for reconciling existing approaches to better collaborate between service providers, consumers, and aggregrators. The discussion of this paper focuses on Web services and their machine-processing service contracts.

The rest of this paper is organized as follow: Section 2 gives an overview of service contracts. The complexity of current contractual concerns is discussed in 3. Guidelines for reconciliation of service contracts are presented in Section 4. We present our preliminary reconciliation results in Section 5. Section 6 concludes the paper.

\section{Overview and Related Work of Service Contracts}

A contract is any formal agreement between two or more involved parties. A contract is a legally binding exchange of promises or agreement between parties that the law will enforce [2]. The contents of contracts may vary as the definition of contract is very broad in scope. In general, a contract includes offer, acceptance of the offer, consideration and an intention to create legal relations ${ }^{3}$. In general, the making of a service contract consists the following phases.

- Specify contract terms: Service providers and/or consumers can specify their contractual terms. Contract terms are fundamental aspects for service contracts. They basically describe the associated properties of a service. These properties may cover QoS, business aspects, legal, and intellectual property issues related to the service usage. A contract term is basically a constraint on a service property, for example, cost is lesser than 5 Euros.

- Negotiate and establish contract: During this phase, contractual terms are negotiated and established between providers and consumers. In some specific situations, renegotiation of contracts are allowed.

- Monitor and enforce the contract: In this phase, an agreed contract is monitored and enforced. A set of metrics for measuring and evaluating contract terms are considered by providers and/or consumers.

There exist many existing works related to service contracts. We analyzed major existing works, including the Web Service Level Agreement (WSLA) framework [4], WSAgreement [5], SLAng [6], WSOL [7], WS-Policy [8], Web Services Policy Language (WSPL) [9], OASIS ebXML Collaboration Protocol Profile (CPP) and Collaboration Protocol Agreement (CPA) [10], ODRL-S [11], and WSMO [12]. Table 1 summarizes

\footnotetext{
${ }^{3}$ In civil law systems [3], the concept of consideration is not central in contracts.
} 
current support on service contracts with respect to QoS (e.g., performance, security, and dependability terms), business (e.g., financial terms), legal (e.g., jurisdiction place, warranty and limit of liability), and IPR (intellectual property rights, e.g., share alike, commercial distribution, free). As shown in Table 1, existing approaches have addressed only few aspects (concerns and phases) associated with service contracts.

\begin{tabular}{|l|l|l|l|l|l|}
\hline Approaches & QoS & Business & Legal & IPR & Phases \\
\hline WSLA[4, 13] & + & + & & & specification, monitoring \\
\hline WS-Agreement [5] & + & + & + & & specification, monitoring, negotiation \\
\hline SLAng & + & + & & & specification, monitoring \\
\hline WSOL[7] & + & + & + & & specification, negotiation \\
\hline WS-Policy[8] & + & & & & specification \\
\hline WSPL[9] & + & & & & specification \\
\hline ebXML CPP/CPA[10] & & + & & & specification \\
\hline ODRL-S[11] & + & + & + & + & specification \\
\hline$W S M O[12]$ & + & & & & specification \\
\hline
\end{tabular}

Table 1. Current support on contractual terms.

\section{Complexity of Current Contractual Concerns}

With the illustrative scenario inspired by the COMPAS project ${ }^{4}$, shown in Figure 1, we intend to give an impression of the complexity of contractual terms of today's interorganizational Web services and the requirement for reconciliation of contractual concerns. Assume that each Web service has a provisional contract (one of the terms in those contracts are represented by $\left\{C_{X S}\right\}$ which has an impact from a given policy tagged with the service) and a contract (represented as $C_{X Y}$ diagrammatically in a shaded square) is established by negotiation between two Web services. The process flow is represented by arrowed dotted dash lines and contractual negotiations and establishments are represented by arrowed black lines.

We are considering a supply chain management scenario which involves multiple Web services (possibly from different organizations) collaborating with each other. A Request Service issuing a purchase request to a Purchase Order Service. The Purchase Order Service has a provisional contract that includes a intellectual right policy on information represented in $\left\{C_{P S}\right\}$. The order sent by this service is being processed by a Purchase Processing Service which has a provisional contract $\left\{C_{A S}^{\prime}\right\}$. These contracts are negotiated and agreed upon the terms defined as $C_{P A}$.

Assume that a security-related QoS policy has been associated with the Purchase Processing Service which has its impact on the provisional contract in $\left\{C_{A S}\right\}$. When the Purchase Processing Service interacts with the provisional contract $\left\{C_{F S}^{\prime}\right\}$ of $F i$ nancial Service, a new contract is established as $C_{A F}$.

\footnotetext{
${ }^{4}$ http://www.compas-ict.eu
} 
A Shipping Service processes the Payment Service by establishing a contract $C_{F T}$, satisfying contractual terms of $\left\{C_{F S}\right\}$ and $\left\{C_{A S}^{\prime}\right\}$. The Shipping Service gives certain information about the transferred goods to a Purchase Verification Service by mutual contracts establishment. Similarly, the Purchase Verification Service sends the verified information about the goods ordered to the Request Service, to complete the process of requisition of order to receiving of goods.

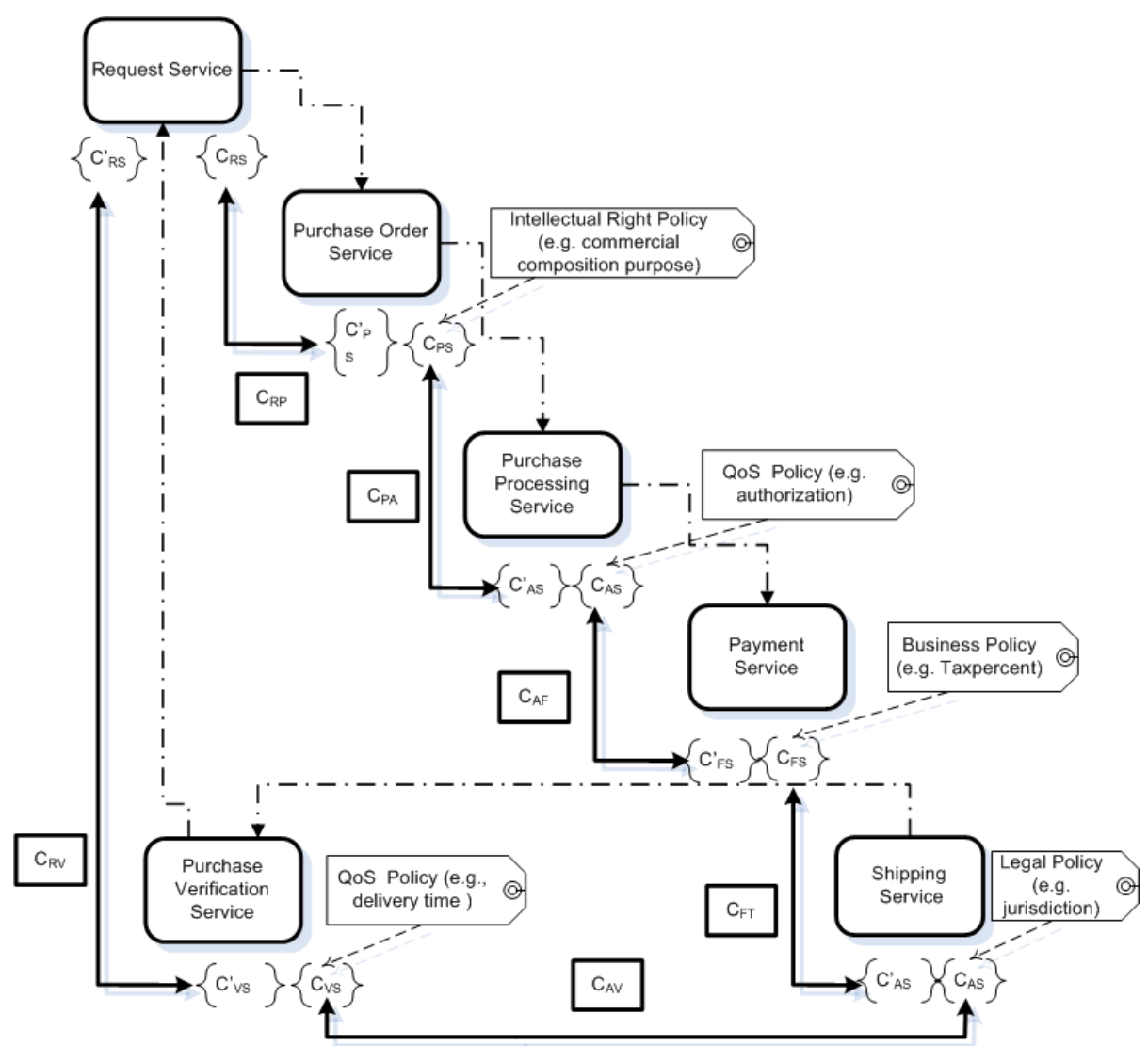

Fig. 1. Complexity of contractual concerns in Web services - a scenario based on COMPAS supply chain management

The illustrated scenario shows a high level of complexities in representing contracts for inter-organizational services. The differences in policy properties and their representations make the scenario complex. First, the current non-uniformity in policy properties will make how to represent policy properties so that they can interact. For example, in our scenario, Payment Service specifies business policy - including financial terms - in $\left\{C_{F S}\right\}$. As today there is no common ontology for properties of a contract, financial terms can be represented in the set of quality policy by Shipping Ser- 
vice. Second, as these properties can be represented in several XML-based languages, there exists several silos between contracts, SLAs, licenses, and policies. Consider that Payment Service represents the provisional contract in the WSLA framework and Purchase Processing Service represents the provisional contract in the SLAng. In this case, there are no defined regulations on how these contracts can communicate and establish a new contract. While some works, such as [13], proposed a solution for managing contractual terms across organizations, they mostly assume that all organizations use the same language/specification in describing contractual terms. Here we further examine cases in which organizations use different languages to model the contractual terms.

\subsection{Issues on Contract Specification in Web Services}

For performing any kind of business, some form of understanding is required between a producer (seller) and a consumer (buyer). This mutual understanding about the business transaction is established by several approaches across various application domains. Some of the most common approaches are specifying contracts, policies, licenses, and service level agreements. Contracts can specify technical aspects (for example, network reliability or availability), business aspects (for example, price), and legal aspects (for example, indemnifications or limitation of liabilities).

Policies establish a relationship between involved parties, specifying obligations and authorizations. Obligations specify the set of activities that an object must or must not perform on target objects and authorizations specify the set of activities that an object is permitted or prohibited to perform on target objects [14].

A Service Level Agreement (SLA) contains technical data relating to the operation of services that implies the objectives with regard to a service consumer [15]. A SLA, e.g. described by WSLA or WS-Agreement specifications, is a bilateral statement signed between a service provider and a service consumer, over the agreed terms and conditions of the given service. A SLA describes the minimum performance criteria a provider promises to meet while delivering a service and typically sets out the remedial action and any penalties that take effect if performance falls below the promised standard. Thus, a SLA specifies the expected operational characteristics of a service in business oriented terms between a provider and a consumer, so that the characteristics can be measured, monitored, and managed $[16,17]$. A SLA is often custom-made and negotiated between a consumer and a provider of a service. Templates for SLA specifications may vary with service providers. SLA negotiation can be a complex process as several terms and conditions related to performance criteria may involve in a SLA.

Policies and licenses are similar in that they govern what a service does, but are not the same. Policies provide the means for specifying and modulating the behavior of a feature to align its capabilities and constraints with the requirements of its users [18] where as service licenses reflect the rights of the providers to control how the service is distributed. A service license primarily focuses on the usage and provisioning terms of services. Being the mechanism of technology transfer [19], service licensing is the method of getting financial benefits for the providers. Optionally, a service license can include SLA terms. Thus, a service license can be broader than a SLA, protecting the rights of service providers and service consumers. The agreement between the service 
provider and a consumer is bound to comply with license clauses, but in general, a license itself is not part of the negotiation.

In our illustrated scenario, as different organizations are participating, these organizations could term contractual concerns in different ways. Though there are some philosophical differences exist among contracts, policies, SLAs, and licenses, the common denominator is an identification for a belief of a business transaction between the service provider and the service consumer and thereby, commonly considered under the umbrella term 'contractual concerns'.

The properties of a contract can describe quality of service aspects (response time, availability, security policy, etc.), legal issues (fair use, copyrights, etc.), intellectual property policies (allowing or denying composition), and business policies (payment, tax, etc.). As there is no formal ontology or unique way of describing these properties, services can represent these properties as they wish. This will cause redundancies in properties description.

In the world of Web services, Web service Description Language (WSDL) [20] is the standard way to describe what a service does. From a given WSDL specification, the location of the service, the protocols supported by the service, and the functionalities provided in terms of incoming and outgoing messages can be known. Research focusing on languages to enhance and to complete the description provided by WSDL are continually in progress. These languages/standards are mostly complementary to WSDL address contractual concerns with varying levels of details.

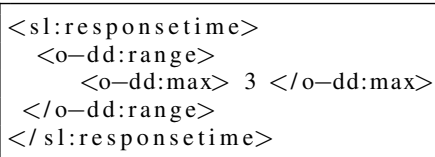

Fig. 2. Example of QoS in ODRL-S

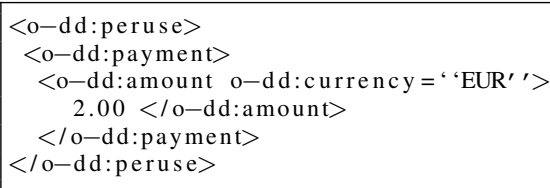

Fig. 4. Example of financial terms in ODRL-S.

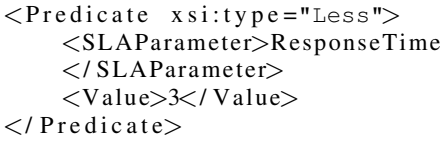

Fig. 3. Example of QoS expressions in WSLA

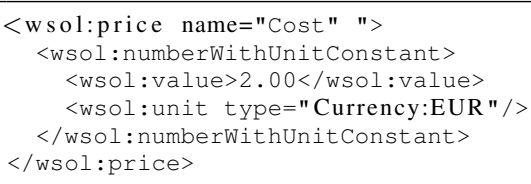

Fig. 5. Example of financial terms in WSOL

Now, we analyze the complexities in representing contractual terms in the said different languages with respect to our illustrated scenario. Quality of service issues of Web services can be expressed in SLAng, WSOL, WSLA, WS-Agreement or ODRLS. Financial policy can be expressed in WSOL, ODRL-S, or WSLA. Examples of the same QoS and financial terms described in different approaches are given in Figures 2 and 3 and Figures 4 and 5, respectively. Even the same policies are expressed in different languages between different Web services, there will be problems in interpretations of those policies by Web services. When the given provisional contracts of two 
Web services interact to establish a contract, there arises misinterpretations due to nonunified/nonstandard approaches in expressing contractual terms of services. Assume that the provisional contract of Payment Service is specified in ODRL-S in Figure 2. Assume that Purchase Processing Service specifies QoS in WSLA in Figure 3. Though these two services specify similar quality of service issues, there would be problems in automatic negotiations and composition of these services because these provisional contracts differ in language semantics and syntax.

\subsection{Issues on Contract Negotiation and Establishment}

In a business environment, a service provider specifies certain contractual terms which describe functional and/or non-functional properties of services. Service consumers also specifies their expectation about functional and/or non-functional properties of their requested services. In a win-win business transaction, a provider and a consumer should agree upon contractual terms by negotiation and thereby establishing a contract.

Price, QoS specifications, and warranties are negotiable terms of a contract. Generally, consumers negotiate on these aspects with providers, using pre-defined templates. Negotiation refers to the comprising exchanges of offers and requests between the participating actors. Theoretical bases of SLA negotiation are provided in [21], identifying the requirements of a negotiation support system. A common understanding for the contractual terms among the negotiating parties is a critical issue in negotiation. To resolve this ontological issue in [22], template based negotiation is suggested [23]. The Policydriven Automated Negotiations Decision-making Approach (PANDA) [24] automates the process of decision making within negotiation.

In case of the illustrated supply scenario, there are policies (such as legal policies like "non-commercial use") which cannot be measured by any means. However, these policies are significant in establishing a contract.

\subsection{Issues on Contract Monitoring and Enforcement}

Consider a scenario where a service consumer is required to pay for a use of particular service through credit card. A message would be delivered to the service provider about payment from the consumer. However, unless the credit card consortium informs about transaction, the provider cannot know whether it is paid and the amount of money paid for an use of a service by consumer. Business term, being one of the important contractual terms, can be measured through an approach involving a third party who informs to provider on the moment of payment by a consumer.

In general, many QoS terms are measurable. QoS terms can be measured at the consumer side to confirm whether the received QoS is in conformance with the QoS offered by providers. There can be contradictions in measurement claiming the differences when a single QoS term is measured at both consumer side and provider side. There should be a consensus in measuring and monitoring of QoS and should be specified in a contract in indemnification clauses. However, there are some contractual terms which are difficult to monitor, for example, non-commercial use ${ }^{5}$.

\footnotetext{
${ }^{5}$ A service provider can allow the service to be used for commercial use or non-commercial use. An 'allowable non-commercial user' [25] of a service will be an atomic or composed service
} 
Most of the works in the area of service contracts focus on specifying and establishing contracts. The area of monitoring and enforcement of service contracts is given very less attention by the community and is focused mainly QoS terms and partially business terms for individual consumer-to-provider scenarios, not for cross-organizational scenarios, e.g. as described in Section 3. Current proposals on measuring contractual terms are summarized as follows:

\begin{tabular}{|l|l|l|}
\hline Contractual Terms & Methods of Measurement & Active Party \\
\hline QoS terms & $\begin{array}{l}\text { Sampling at regular intervals of time, querying through di- } \\
\text { rect communication between provider and consumer }\end{array}$ & $\begin{array}{l}\text { Consumer, } \\
\text { Provider }\end{array}$ \\
\hline Business terms & Notification through trusted third party approved services & Provider \\
\hline
\end{tabular}

In the pioneering work of WSLA [4], the way of specifying, measuring, and monitoring the SLA parameters are described. Upon receipt of an SLA specification, the WSLA monitoring services are automatically configured to enforce the SLA. An event calculus based approach for monitoring SLAs in a utility computing scenario is presented in [26]. The recent work in [27] focuses on determining elements of SLAs that are monitorable at which degree. However, none of these approaches are sufficient to monitor completely contractual terms described by different specifications and associated with different services and to make decision strategies based on the outcome of monitoring.

\section{Towards Reconciliation and Further Investigation}

We have explored several specifications for representing contractual concerns. Many of the contract terms are different but yet represent the same thing. We have identified the following key research questions: Can we have a single language to represent all contractual concerns? Generally, all specification languages/standards focus on terms and conditions to be agreed by providers and consumers. Every language describes certain properties of services entirely. Unfortunately, today, there is not a single language that fully supports all contractual terms. In the existence of multiple languages to represent contractual concerns, a unified set of standards for contractual terms is needed. To deal with multiple specifications, we can start from the consumer's point of view: we should provide a common language for the consumer to specify the requesting contractual terms. Such a common language can be used together with common ontologies for business, legal and intellectual terms. Based on consumer-specified contractual terms, we apply data integration techniques, such as schema mapping, meta-model and domain-specific languages, to query, evaluate and compare contractual terms given by different specifications/languages. Some have proposed the combination of different approaches, such as WSMO with WS-Policy [28], but the support of consumer-specified service contracts has not been focused.

consumer or provider serving for non-profit as defined under the copyright law of respective jurisdictions. The use of a service by individuals, educational institutions or nonprofit organizations are regarded as non-commercial use. When a service uses another service that includes the non-commercial use clause, the consuming service is expected not to violate the clause of non-commercial use. 
Though there is no unified language, do the existing languages/standards satisfy the requirement of consumers for representing contractual concerns? Interestingly, most of the present languages describing contractual concerns fail to represent hierarchical user preferences in contracts. For example, consider the following scenario where a consumer is interested in consuming a service with lesser cost and better response time. The consumer wishes to specify the order of preferences for the service to be consumed as follows. The consumer considers price as a priority term, i.e. for less price service, the consumer wants to select a service in which cost is lower followed by higher response time. The present languages describe these preferences in a segregated manner and policy-aware service selection algorithms handle these scenarios up to a certain threshold. The cause today that the language not describing preferences by tree based data structure is the level of depth for representing contractual terms by these languages. Mostly, these languages express contractual terms in a single level. Representing contractual terms in a hierarchical form (e.g., by a tree data structure) would be one simple solution but can enhance the description of consumer preferences and enable algorithms working on contractual terms to be more efficient. In this sense, the concept of constraints hierarchies [29] can be used to select services [30].

Can we manage contracts associated with service instances separately from WSDL? Existing tools tend to assume that contracts can be annotated with service description to facilitate the service discovery. However, a contract, if deployed in real business, will be associated with service instances. In particular, the concept of SaaS introduces various challenges to manage contracts associated with services because each customer, in principle, would have a different contract for each service instance.

Can we use different contract negotiation/ compatibility algorithms, even though we do not have a unified approach? Presently, negotiation is mainly a manual process, and thus a full or partial automated contract negotiation is needed. Furthermore, in the case of composing services associated with different contracts, compatibility should be checked. However, this has so far attracted little attention.

Is real time monitoring and enforcement of the contractual concerns, in particular, legal and intellectual right terms, of dynamic Web services possible? Automated contract management and enforcement is highly desirable. It is obvious that contractual terms cannot simply be monitored by the consumer and the provider in a fully automatic fashion, as assumed in most current work. The third party is needed in many cases, as discussed in [13]. We can study the model of joint control in virtual organizations [31] for developing similar service contract authorities. Furthermore, third parties for service contracts monitoring and enforcement must be able to deal with multiple types of contract specifications. In our view, it would be possible to automatically monitor and enforce QoS and business terms at realtime. However, for legal and intellectual right terms, not only third parties are required for contract enforcement but also manual monitoring and enforcement will be required. To support contract monitoring and enforcement in semi-automatic processes, the gap between technical contract terms in business (human processing) level and in operational (machine processing) level, as discussed in [1] has to be addressed. 


\section{Preliminary Results on The Reconciliation}

Addressing the questions in Section 4 will need a lot of effort from Web service community and we have achieved some preliminary results on the reconciliation of service contracts. To support the management of service contracts, we have developed SEMF (Service Evolution Management Framework) [32] which can be used as a platform for service contract-based SaaS. In SEMF, service licenses/contracts can be described by different languages, such as ODRL-S, WS-Policy or WS-Agreement, and are managed separately from other information, such as WSDL, as given in an example in Listing 1.1. Thus, SEMF can serve as a contract registry for complex business scenarios.

To support the establishment of contractual concerns described by different specifications, we first define a simple schema for representing consumer-specified contracts (see Figure 6). From the consumer-specified contracts, we apply data mapping and integration techniques to query and map different types of contract information provided by different services. For example, by utilizing XQuery and SPARQL, we can query contract terms described by XML, RDF and OWL-based specifications and stored in SEMF, and map them to the consumer-specified contract model. Based on that, we perform the contract search and compatibility analysis.

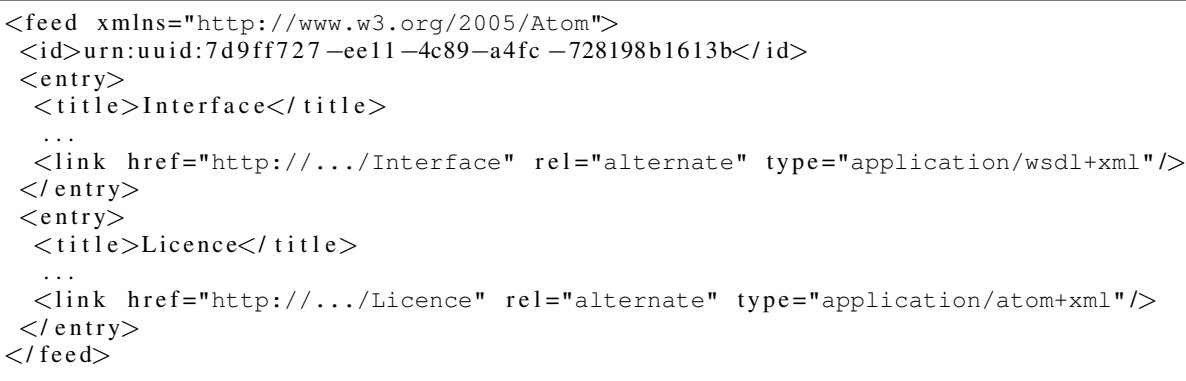

Listing 1.1. Example of a feed describing License and Interface

\section{Conclusion}

In a dynamic market environment, the usage of Web services is based on contracts specifying the terms and conditions of using and provisioning of services. This paper discusses the strengths and weakness of existing languages and standards for describing contracts in service oriented computing. When the vision of software as a service is realized, service contracts are of paramount importance and we need to consider the interoperability of service contracts provided by different organizations. Based on our study, we have suggested some guidelines to reconcile the contractual concerns of Web services that could make the vision of service oriented computing more realistic. Our future work is to tackle some open issues identified in this paper.

\section{References}

1. Arenas, A., Wilson, M.: Contracts as trust substitutes in collaborative business. Computer 41(7) (2008) 80-83 


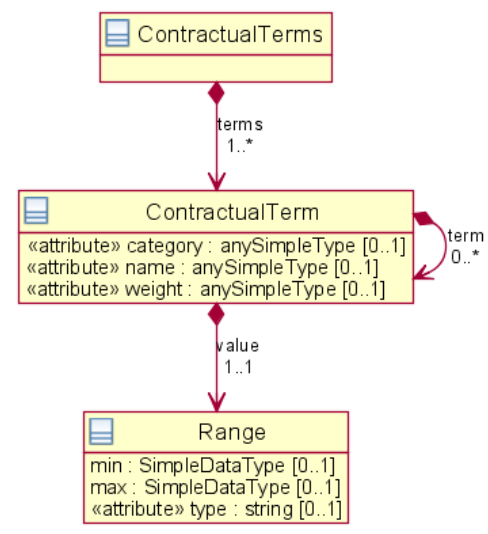

Fig. 6. Simplified model for consumer-specified service contracts

2. Wehberg, H.: Pacta Sunt Servanda. The American Journal of International Law 53(4) (1959)

3. Apple, J., Deyling, R.: A Primer on the Civil Law System. Federal Judicial Center, USA (1995)

4. Keller, A., Ludwig, H.: The WSLA Framework: Specifying and Monitoring Service Level Agreements for Web Services. Journal of Network and Systems Management 11(1) (2003)

5. Andrieux, A., Czajkowski, K., Dan, A., Keahey, K., Ludwig, H., Nakata, T., Pruyne, J., Rofrano, J., Tuecke, S., Xu, M.: Web Services Agreement Specification(WSAgreement). Grid Resource Allocation Agreement Protocol (GRAAP) WG, http://www.ogf.org/documents/GFD.107.pdf (2007)

6. Skene, J., Lamanna, D., Emmerich, W.: Precise Service Level Agreements. In: Proceedings of 26th International Conference on Software Engineering (ICSE). (2004)

7. Tosic, V., Pagurek, B., Patel, K., Esfandiari, B., Ma, W.: Management Applications of the Web Service Offerings Language. In: Proceedings of the 15th Conference on Advanced Information Systems Engineering. (2003)

8. Vedamuthu, A., Orchard, D., Hirsch, F., Hondo, M., Yendluri, P., Boubez, T., Yalcinalp, U.: Web Services Policy (WS-Policy) Framework (2007) http://www.w3.org/TR/ws-policy.

9. Anderson, A.: Web Services Policy Language (WSPL). In: Proceedings of the Fifth IEEE International Workshop on Policies for Distributed Systems and Networks (POLICY'04). (2004) 189-192

10. OASIS ebXML CPP and CPA Technical Committee: Collaboration Protocol Profile and Agreement Specification Version 2.1. http://www.oasis-open.org/committees/ebxml-cppa/ (2005)

11. Gangadharan, G.R., D'Andrea, V., Iannella, R., Weiss, M.: ODRL Service Licensing Profile (ODRL-S). In: Proceedings of the 5th International Workshop for Technical, Economic, and Legal Aspects of Business Models for Virtual Goods. (2007)

12. Toma, I., Foxvog, D., Jaeger, M.C.: Modeling qos characteristics in wsmo. In: MW4SOC '06: Proceedings of the 1st workshop on Middleware for Service Oriented Computing (MW4SOC 2006), New York, NY, USA, ACM (2006) 42-47

13. Keller, E., Ludwig, H.: Defining and monitoring service-level agreements for dynamic ebusiness. In: LISA '02: Proceedings of the 16th USENIX conference on System administration, Berkeley, CA, USA, USENIX Association (2002) 189-204

14. Lupu, E., Sloman, M.: A Policy Based Role Object Model. In: Proceedings of the International Enterprise Distributed Object Computing Conference (EDOC). (1997) 
15. Muller, N.: Managing Service Level Agreements. International Journal of Network Management 9 (1999) 155-166

16. Lewis, L., Ray, P.: Service Level Management Definition, Architecture, and Research Challenges. In: Proceedings of the Global Telecommunications Conference (GLOBECOM). (1999)

17. Sahai, A., Durante, A., Machiraju, V.: Towards Automated SLA Management for Web Services. Technical Report HPL-2001-310 (R.1), Software Technology Laboratory, HP Laboratories, Palo Alto, USA (2002)

18. Kamoda, H., Yamaoka, M., Matsuda, S., Broda, K., Sloman, M.: Policy Conflict Analysis Using Free Variable Tableaux for Access Control in Web Services Environments. In: Proceedings of the 14th International World Wide Web Conference (WWW). (2005)

19. Speser, P.: The Art and Science of Technology Transfer. John Wiley \& Sons, USA (2006)

20. Christensen, E., Curbera, F., Meredith, G., Weerawarana, S.: Web Services Description Language (WSDL) 1.1. http://www.w3.org/TR/wsdl (2001)

21. Demirkan, H., Goul, M., Soper, D.: Service Level Agreement Negotiation: A Theory-based Exploratory Study as a Starting Point for Identifying Negotiation Support System Requirements. In: Proceedings of the 38th Hawaii International Conference on System Sciences. (2005)

22. Michael, S.: Engineering Electronic Negotiations. Kluwer Academic Publishers, New York, USA (2002)

23. Grosof, B., Reeves, D., Wellman, M.: Automated Negotiation from Declarative Contract Descriptions. Computational Intelligence 18(4) (2002)

24. Gimpel, H., Ludwig, H., Dan, A., Kearney, B.: PANDA: Specifying Policies for Automated Negotiations of Service Contracts. In: Proceedings of the First International Conference on Service Oriented Computing. (2003)

25. CreativeCommons: Proposed Best Practice Guidelines to Clarify the Meaning of "Noncommercial" in the Creative Commons Licenses. http://wiki.creativecommons.org/DiscussionDraftNonCommercial_Guidelines (Accessed on August, 2007)

26. Farrell, A., Sergot, M., Salle, M., Bartolini, C.: Using the Event Calculus for Tracking the Normative State of Contracts. International Journal of Cooperative Information System 4(2) (2005)

27. Skene, J., Skene, A., Crampton, J., Emmerich, W.: The monitorability of service-level agreements for application-service provision. In: WOSP '07: Proceedings of the 6th international workshop on Software and performance, New York, NY, USA, ACM (2007) 3-14

28. Kopecky, J., Roman, D.: D24.3v0.1 aligning wsmo with ws-policy (2007) WSMO Working Draft, http://wsmo.org/TR/d24/d24.3/v0.1/.

29. Borning, A., Freeman-Benson, B.N., Wilson, M.: Constraint hierarchies. Lisp and Symbolic Computation 5(3) (1992) 223-270

30. Guan, Y., Ghose, A.K., Lu, Z.: Using constraint hierarchies to support qos-guided service composition. In: ICWS, IEEE Computer Society (2006) 743-752

31. Smith, T., Ramakrishnan, L.: Joint policy management and auditing in virtual organizations. grid 00 (2003) 117

32. Treiber, M., Truong, H.L., Dustdar, S.: SEMF - Service Evolution Management Framework. In: 34th EUROMICRO Conference on Software Engineering andAdvanced Applications (SEAA), Special session on Quality and Service-Oriented Applications, IEEE Computer Society (2008) 